\title{
Universality in fully developed turbulence
}

\author{
Siegfried Grossmann ${ }^{1}$ and Detlef Lohse ${ }^{1,2}$
}

October 29, 2018

\author{
1 Fachbereich Physik, Philipps-Universität, \\ Renthof 6, D-35032 Marburg, Germany \\ 2 The James Franck Institute, The University of Chicago, \\ 5640 South Ellis Avenue, Chicago, IL 60637, USA
}

\begin{abstract}
We extend the numerical simulations of She et al. [Phys. Rev. Lett. 70, 3251 (1993)] of highly turbulent flow with $15 \leq$ Taylor-Reynolds number $R e_{\lambda} \leq 200$ up to $R e_{\lambda} \approx 45000$, employing a reduced wave vector set method (introduced earlier) to approximately solve the Navier-Stokes equation. First, also for these extremely high Reynolds numbers $R e_{\lambda}$, the energy spectra as well as the higher moments - when scaled by the spectral intensity at the wave number $k_{p}$ of peak dissipation - can be described by one universal function of $k / k_{p}$ for all $R e_{\lambda}$. Second, the ISR scaling exponents $\zeta_{m}$ of this universal function are in agreement with the 1941 Kolmogorov theory (the better, the large $R e_{\lambda}$ is), as is the $R e_{\lambda}$ dependence of $k_{p}$. Only around $k_{p}$ viscous damping leads to slight energy pileup in the spectra, as in the experimental data (bottleneck phenomenon).
\end{abstract}




\section{Introduction}

\section{$1.1 \quad$ Universal turbulent spectra}

Very high Reynolds number turbulence still resists full numerical simulations. While in experiments Taylor-Reynolds numbers $R e_{\lambda}$ up to $R e_{\lambda}=13000$ have been reported [1], the most turbulent numerical flow has - to our knowledge - been realized by She et al. [2], who achieve $R e_{\lambda}=200$ at a resolution of $512^{3}$. These authors find that for all $R e_{\lambda}$ up to $R e_{\lambda}=200$ all their energy spectra coincide when scaled by the spectral intensity at the wave number $k_{p}$ of peak dissipation. I.e., the function

$$
\left\langle|\mathbf{u}(\mathbf{k})|^{(2)}\right\rangle /\left\langle\left|\mathbf{u}\left(\mathbf{k}_{p}\right)\right|^{2}\right\rangle=F^{(2)}\left(k / k_{p}\right),
$$

is universal as assumed by Kolmogorov and Obukhov [3] - both in the inertial subrange (ISR) and in the viscous subrange (VSR). Note that $F^{(2)}(1)=1$ by definition. The universality is also found in experimental spectra [4]. For further numerical simulations, see also [5].

Kolmogorov and Obukhov [3] not only assumed universality of $F^{(2)}\left(k / k_{p}\right)$, but also its power law behavior $F^{(2)}\left(k / k_{p}\right) \propto\left(k / k_{p}\right)^{-\zeta_{2}}$ in the ISR with the classical exponent $\zeta_{2}=2 / 3$. (The scaling exponent $2 / 3$ in the discrete Fourier transform, which we use here, corresponds to $5 / 3$ in the continuous case.)

It has been argued in a long lasting debate (see e.g. [6, 0, 8]) that there are small intermittency corrections to the classical scaling exponent $\zeta_{2}=2 / 3$. Unfortunately, even at today's state of the computational art [2], $\zeta_{2}$ cannot sufficiently precisely be determined from full numerical simulations so that one could confirm or rule out deviations from $2 / 3$. The reason is that for the tractable $R e_{\lambda}$ the available wave number range is quite narrow $\left(k_{p} / k_{\min } \approx 5\right.$ in [2]; $k_{\text {min }}$ is the lowest wave number free of forcing). To obtain an ISR which extends over more than a decade, $k_{p}$ must be larger than $50 k_{\min }$ [2]. To realize this, a resolution $\geq 1500^{3}$ is required [2]. The required computer work increases as $R e_{\lambda}^{6} \log _{2} R e_{\lambda}$ [9]. We are thus far away from being able to create developed turbulence in a numerical flow for as high $R e_{\lambda}$ as in experiment [1]. The huge gap between experiments and simulations is demonstrated in table 1. We therefore still need reasonable approximation techniques to numerically solve the Navier-Stokes equation.

\subsection{Reduced wave vector set approximation for high $R e$ - turbulence}

Such an approximation has been introduced by us in [10, 11]. Meanwhile we could considerably improve our approach [12]. Here we employ it to study universal features of fully developed turbulence for Reynolds numbers Re between 730 and $1.4 \cdot 10^{7}$ (corresponding to Taylor-Reynolds numbers $R e_{\lambda}$ between 120 and 45000 , see table 2 below). This leads to remarkably large ISRs. For $R e=1.4 \cdot 10^{7}$, the extension of 
the ISR is $k_{p} / k_{\text {min }} \approx 2000$, i.e., more than three decades, compared to $k_{p} / k_{\text {min }} \approx 5$ achieved in the presently best Navier-Stokes simulation [2].

For completeness we shortly repeat the main idea of our approximation. To deal feasibly with the many scales present in turbulent flow, we only admit a geometrically scaling subset $K$ of wave vectors in the Fourier sum, $K=\cup_{l} K_{l}$, thus $u_{i}(\mathbf{x}, t)=$ $\sum_{\mathbf{k} \in K} u_{i}(\mathbf{k}, t) \exp (i \mathbf{k} \cdot \mathbf{x}) . K_{0}=\left\{\mathbf{k}_{n}^{(0)}, n=1, \ldots, n_{\max }=80\right\}$ contains appropriately chosen wave vectors, $K_{l}=\left\{\mathbf{k}_{n}^{(l)}=2^{l} \mathbf{k}_{n}^{(0)}, \quad n=1, \ldots, n_{\max }=80\right\}, l=1, \ldots, l_{\text {max }}$, are scaled replica of $K_{0}$. The choice of $l_{\max }$ depends on the control parameter $\nu$, the viscosity. The incompressible Navier-Stokes equation is solved on $K$ with periodic boundary conditions in a box of size $(2 \pi L)^{3}$. All lengths will from now on be measured in multiples of $L$, so the smallest component of the smallest wave vector is 1 . The flow is permanently, non-stochastically forced on the outer length scale with energy input rate $\epsilon$. All times will henceforth be given in multiples of $\left(L^{2} / \epsilon\right)^{1 / 3}$, i.e., the energy input and thus in the stationary case also the energy dissipation rate is $\epsilon=1$. The type of forcing does not influence our results sizeably [12]. The smallest wave vectors whose amplitudes are free of forcing have length $k_{\min }=3$. The smallest wave vectors at all are $\pm(1,1,-2)+$ permutations and thus have length $\sqrt{6}=0.82 k_{\text {min }}$. All wave vector amplitudes $\mathbf{u}(\mathbf{k}, t)$ with $|\mathbf{k}| \geq k_{\text {min }}=3$ are free of forcing.

\subsection{Contents of the paper}

The paper is organized as follows: In section 2 we examine the $R e$-dependence of the Taylor-Reynolds number. In section 3 we confirm universality up to the highest $R e$ we can treat. The form of the universal spectra is discussed in section 4 . Section 5 is left for a short summary of our findings.

\section{Re-dependences}

We now come to our results. First, what are the Reynolds numbers $R e$ of our approximate numerical turbulence? As usual, there is some arbitrariness in the definition of the Reynolds number. We regard $L_{0}=\lambda / 2$ as the outer length scale, where $\lambda$ is the wave length of the smallest wave vector, and $U_{0}=2 u_{1, r m s}$ as the typical velocity difference on the outer lengthscale. Thus $R e=U_{0} L_{0} / \nu$ can be considered as an appropriate definition of the Reynolds number. The data for five simulations, covering four decades of $R e$, are given in table 2 . We also list the Taylor Reynolds numbers $R e_{\lambda}=u_{1, r m s} \lambda_{\text {Taylor }} / \nu$, where $\lambda_{\text {Taylor }}=u_{1, r m s} /\left(\partial_{1} u_{1}\right)_{r m s}$ are the Taylor lengths.

The dissipation rate is balanced by the input rate $\epsilon \sim U_{0}^{3} / L_{0}$ [13, [1]. We therefore write

$$
\epsilon=c_{\epsilon} U_{0}^{3} / L_{0},
$$

where $c_{\epsilon}$ is a dimensionless number. Since we choose $\epsilon=1, L_{0}=\pi / \sqrt{6}$, and since 
we find $U_{0}=2 u_{1, r m s}$ from the numerical solution, we can determine $c_{\epsilon}$ from this equation. It turns out to decrease with increasing $R e$, seemingly to a final level somewhere near $6 \cdot 10^{-3}$. Note that for laminar flow, on the other hand, it holds $c_{\epsilon} \propto R e^{-1}$, see e.g. [13].

Equation (2) leads to the relation

$$
R e_{\lambda}=\sqrt{\frac{15}{16 c_{\epsilon}}} \sqrt{R e} .
$$

When $c_{\epsilon}$ eventually becomes universal, i.e., independent of $R e$, the well known large $R e$ limit relation $R e_{\lambda} \propto \sqrt{R e}$ is recovered. In experiment, for smaller $R e$ the measured Taylor Reynolds number $R e_{\lambda}$ turns out to be smaller than predicted by $R e_{\lambda} \propto \sqrt{R e}$ [15, 16], as in fact $c_{\epsilon}$ depends on $R e$. Both the large $R e$ behaviour of $R e_{\lambda}$ with the power law exponent $1 / 2$ and the deviations for smaller $R e$ can also be seen in our approximate solutions, see Fig. 1. For a more detailed discussion on the $R e$-dependence of $c_{\epsilon}$ and $R e_{\lambda}$, see [17, 18].

One remark concerning the nominal value of the prefactor in eq. (3). Taking the large Reynolds numbers, we have (with $c_{\epsilon}=6.5 \cdot 10^{-3}$ from table 2 ) $R e_{\lambda} \approx 12 \sqrt{R e}$, whereas experimentally it is $R e_{\lambda} \sim \sqrt{R e}$, i.e., we overestimate the Taylor Reynolds numbers by one order of magnitude. We explain this as due to our approximation, as in our reduced wave vector set $K$ the larger wave vectors are considerably thinned out. So less energy than ought to be is transported downscale, leading to a larger $u_{1, r m s}$ than in real turbulence and thus to a larger $R e_{\lambda}=u_{1, r m s}^{2} / \nu\left(\partial_{1} u_{1}\right)_{r m s}$. The same blocking effect by phase space sparseness at larger $k$ also leads to an overestimation of the Kolmogorov constant $b$ in the velocity structure function $D(r)=b(\epsilon r)^{2 / 3}$ as already mentioned in [12, 11] - also by a factor of about $10(b \approx 70$ instead of $b=8.4$ as in experiment, see [12]), because both $R e_{\lambda}$ and $D(r)$ are quadratic in

$u_{1, r m s}$. (Note that the denominator $\left(\partial_{1} u_{1}\right)_{r m s}$ is proportional to $\sqrt{\epsilon}$ and thus kept fixed.)

\section{Universal wave number spectra}

The wave number spectra of our approximate solutions are shown in Fig. 2. We can confirm that $F^{(2)}\left(k / k_{p}\right)$ in fact is universal for all $R e$ even much beyond $R e_{\lambda}=200$ as studied in [2]. As in [2], the wave number $k_{p}$ of peak dissipation is found to increase as $R e^{3 / 4}$, i.e., $\eta k_{p}$ is constant also for the huge $R e$ we simulated, see table 3. We find $k_{p} \approx 1 / 10 \eta$. For $R e=1.4 \cdot 10^{7}$ the ISR extends as far as 3.3 decades to the left of $k_{p}$, see table 2 . In k-space $\log _{10}\left(k_{p} / k_{\text {min }}\right)$ gives the extension of the ISR. This extension of the scaling range should also follow from the Reynolds number $R e$. In r-space the scaling occurs between the outer length scale $L_{0}$ and $10 \eta$, where $\eta=\nu^{3 / 4} / \epsilon^{1 / 4}$ is the Kolmogorov length scale [13, 14]. For large $R e$ it is [13]

$$
L_{0} / 10 \eta=c_{\eta}^{\prime} R e^{3 / 4}
$$


Formally eq. $\left(4^{\prime}\right)$ can be derived from eq. (2) and one gets $c_{\eta}^{\prime}=c_{\epsilon}^{1 / 4} / 10$. Note that thus $c_{\eta}^{\prime}$ is also slightly $R e$-dependent via $c_{\epsilon}(R e)$. Yet our $c_{\epsilon}$ is smaller than the experimental one, see last paragraph of section 2 . Therefore we rather define a $c_{\eta}$ from the extension of the scaling range found in our numerical simulation than from $\left(4^{\prime}\right)$, namely by

$$
k_{\text {min }} / k_{p}=c_{\eta} R e^{3 / 4} .
$$

This corresponds to $\left(4^{\prime}\right)$ since $L_{0}$ is approximately $1 / k_{m i n}$ and $10 \eta$ is approximately $1 / k_{p}$. More precisely we have $L_{0}=3 \pi /\left(\sqrt{6} k_{\text {min }}\right)$, so $L_{0}^{-1}=0.26 k_{\text {min }}$. The ratio $k_{p} / k_{\text {min }}$ can be extracted from our numerical spectra in k-space. For the largest $R e=$ $1.4 \cdot 10^{7}$ we find $k_{p} / k_{\text {min }}=1950$ and thus obtain $c_{\eta}=\left(k_{p} / k_{\text {min }}\right) R e^{-3 / 4}=8.52 \cdot 10^{-3}$. We now disregard the small $R e$-dependence of $c_{\eta}$ and calculate the extension of the scaling ranges for smaller $R e$ from (团) with $c_{\eta}=8.52 \cdot 10^{-3}$. The results are given in the last column of table 2 and are found in excellent agreement with the length of the scaling ranges seen in our approximate solutions for these smaller Re, see 6 th column of table 2. Only for the smallest $R e$ we find disagreement, as expected, because for $R e=730$ the constant $c_{\eta}$ in eq. (4) can no longer be considered as independent of $R e$.

Moreover, also the $F^{(m)}\left(k / k_{p}\right)=\left\langle|\mathbf{u}(\mathbf{k})|^{m}\right\rangle /\left\langle\left|\mathbf{u}\left(\mathbf{k}_{p}\right)\right|^{m}\right\rangle, \mathrm{m}=3,4,6,8,10$, are found to be universal (see Fig. 2 for $m=6$ ). Due to the very extended ISR we can determine the power law exponents $\zeta_{m}$ of the universal functions $F^{(m)}\left(k / k_{p}\right) \propto$ $\left(k / k_{p}\right)^{-\zeta_{m}}$ rather precise, cf. table 3 . This is still not possible in full simulations, as the universal scaling range is too small, $k_{p} / k_{\text {min }} \leq 5$ [5, 2]. If a power law fit for full simulations is tried nevertheless, one gets scaling exponents $\zeta_{m}$ much smaller than the classical ones, due to the nonuniversal large scale forcing [19.

\section{Form of the universal spectra}

Having shown universality, we now check several fits to the universal spectra $F^{(m)}\left(k / k_{p}\right)$ that we obtained from our numerical data.

\subsection{Power law fit and intermittency}

Firstly, we fitted the spectra for all $R e$ by the two parameter function

$$
F^{(m)}(\tilde{k})=\tilde{k}^{-\zeta_{m}} \exp \left[-(\tilde{k}-1) / \tilde{k}_{d}^{(m)}\right], \quad \tilde{k}=k / k_{p}, \quad \tilde{k}_{d}^{(m)}=k_{d}^{(m)} / k_{p} .
$$

This form has theoretical support [20] and was also successfully used to fit experimental spectra [21, 22]. The crossover between the power law behaviour in the ISR and exponential fall off in the VSR takes place at the wave number $k_{d}^{(m)}$. In table 3 we list the parameters, obtained from a fit in the range $0 \leq k \leq \eta^{-1} / 4$ or $0 \leq k / k_{p} \leq 2.5$, respectively. The fit (5) gives $\zeta_{3}$ rather near, but not exactly equal to 1 as it should be according to Kolmogorov's structure equation [14. This tiny deviation is corrected in table 3 by dividing the scaling exponents obtained from the 
fit (5) by $\zeta_{3}$. The resulting scaling exponents take their classical values $\zeta_{m}=m / 3$ with high accuracy, see table 3.

From the appearance of the classical scaling exponents $\zeta_{m}=m / 3$ one might deduce that there is no intermittency in our signal. This conclusion would not be correct. In fact, for small scales we do observe strong intermittency in the signal [10, 12]. We therefore suggested to introduce local $\zeta_{m}(k)$, defined by local fits of type (5) in the restricted k-ranges $[k / \sqrt{10}, k \sqrt{10}]$ for all $k$, keeping the $k_{d}^{(m)}$ fixed at their global values [12].

The surprising result is shown in Fig. 3. There are large intermittency corrections $\delta \zeta_{m}(k)=\zeta_{m}(k)-m / 3$ at small scales (VSR), only moderate intermittency corrections at large scales (stirring subrange, SSR), but hardly any deviations from classical scaling in the ISR. This astonishing result was extensively discussed already in [12. Here it can be confirmed for a considerably larger Re-range.

In addition, we fitted eq. (5) to our spectra, but now with $k_{d}^{(m)}$ fixed at $k_{d}^{(m)}=$ $2 k_{d}^{(2)} / m, k_{d}^{(2)}=(13.5 \eta)^{-1}$, see below. Again we find only tiny global intermittency corrections, which clearly decrease with increasing $R e$, as predicted by [15, 23]. For $R e=1.05 \cdot 10^{4}$ we find $\delta \zeta_{2}=0.012, \delta \zeta_{6}=-0.058$, going down to $\delta \zeta_{2}=0.002$, $\delta \zeta_{6}=-0.011$ for $R e=1.4 \cdot 10^{7}$, suggesting, that intermittency might be a finite size effect. For details and a theoretical explanation we refer to ref. [24].

We now discuss the crossover scale between ISR and VSR. From the fit (5) we get $\left(k_{d}^{(2)}\right)^{-1} \approx 13.5 \eta$, i.e., the crossover scale is one order of magnitude larger than the Kolmogorov length. This fact has long been known from experiment [14 and theory [25]. From the maximum condition for $k^{2} F^{(2)}(k)$ at $k=k_{p}$ it follows that $\tilde{k}_{d}^{(2)}=1 /\left(2-\zeta_{2}\right)=3 / 4$. In our simulations there are some fluctuations around this value. The reason is that we have discrete wave vectors which are not dense in the VSR. Thus $k_{p}$ can only be determined with limited accuracy, cf. Fig. \&, where the energy dissipation rate $\epsilon(k)=\mathbf{k}^{2}\left\langle|\mathbf{u}(\mathbf{k})|^{2}\right\rangle$ is displayed. Typically the relative k-distances are $\delta k / k \approx 1 / 10$, which corresponds to the $\approx 10 \%$ deviations of $\tilde{k}_{d}^{(2)}$ from 0.75 in table 3 . Of course, to increase the accuracy of $k_{p}$, one could also define it in terms of $k_{d}^{(2)}$ from the global fit (5) with fixed $\zeta_{2}=2 / 3$ to be $k_{p}=4 k_{d}^{(2)} / 3$. The resulting small changes are not visible in Fig. 2.

Where does the crossover from ISR to VSR behaviour take place in higher order moments? From the fit (5) we find $k_{d}^{(m)}=2 k_{d}^{(2)} / m$, i.e., $\tilde{k}_{d}^{(m)}=3 /(2 m)$ to a very high precision [12]. This means that the ISR is considerably smaller for higher order moments, or, to state it differently, higher order moments feel viscosity earlier than lower order moments.

\subsection{Energy pileup in the crossover region between ISR and VSR}

We also applied fits different from (5), as from Fig. 5 it might seem that (5) only badly fits the spectrum in the range around $k_{p}$. The same observation was re- 
ported already by She and Jackson [4] when they determined the universal function $F^{(2)}\left(k / k_{p}\right)$ from experimental data. To improve the fit, they suggested to use the empirical three parameter function

$$
F^{(2)}(\tilde{k})=\frac{\tilde{k}^{-2 / 3}}{1+\alpha}\left(1+\alpha \tilde{k}^{\beta}\right) \exp \left[-(\tilde{k}-1) / \tilde{k}_{d}^{(2) \prime}\right], \quad \tilde{k}=k / k_{p}, \quad \tilde{k}_{d}^{(2) \prime}=k_{d}^{(2) \prime} / k_{p}
$$

and found $\alpha=0.8, \beta \approx 0.7$, i.e., near $k=k_{p}$ the decay of the spectral power is diminished. Their physical interpretation is a pileup of excitation around $k_{p}$, possibly due to coherent vortex structures.

We also tried the fit (6) and found $\alpha \approx 2, \beta \approx 1.8$, and $\tilde{k}_{d}^{(2) \prime} \approx 0.4$, all slightly depending on $R e$. Thus the energy pileup at $k \approx k_{p}$ in our approximate NavierStokes solution seems to be even stronger than in experiment, as we have an additive correction term with a larger exponent. We ascribe the larger correction summand to our approximation: larger wave vectors are more and more sparse, so the dissipative scales cannot acquire the kinetic energy as fast as they should and pile it up around $k_{p}$. Of course, $F^{(2)}\left(k / k_{p}\right)$ does not increase with $k / k_{p}$ as the correction term is strongly damped by $\exp \left(-k / k_{d}^{(2) \prime}\right)$ with $k_{d}^{(2) \prime}$ much smaller as $k_{d}^{(2)}$ before. The energy pileup can also be observed in Fig. 5, where we replotted $F^{(2)}\left(k / k_{p}\right)$ with the two fits eq. (可), where we fixed $\zeta_{2}=2 / 3$, and eq. (6). For $k \approx k_{p}$ the fit (6) is slightly superior to the fit (5). We have to raise doubts that the energy pileup is due to coherent vortex structures, as was speculated in [4, because these are less in our approximation than in experiment, cf. [10], while our pileup is stronger than found in the measured data.

Instead, the energy pileup may possibly be explained by the socalled bottleneck phenomenon 26]. This phenomenon can be described as follows: Imagine a triad Navier-Stokes interaction between the amplitudes $\mathbf{u}\left(\mathbf{k}_{1}\right), \mathbf{u}\left(\mathbf{k}_{2}\right)$, and $\mathbf{u}\left(\mathbf{k}_{3}\right), \mathbf{k}_{1}+$ $\mathbf{k}_{2}+\mathbf{k}_{3}=0, k_{1}<k_{2,3}$, so that $\mathbf{u}\left(\mathbf{k}_{2}\right)$ and $\mathbf{u}\left(\mathbf{k}_{3}\right)$ are already considerably damped by viscosity, in additon to the power law decrease $\propto k^{-2 / 3}$. So the turbulent energy transfer downscale $\sim k_{1} u\left(k_{1}\right) u\left(k_{2}\right) u\left(k_{3}\right)$ would be reduced and stationarity could not be achieved, if $\mathbf{u}\left(\mathbf{k}_{1}\right)$ did not increase, i.e., an energy pileup at $k_{1}$ is established. The effect is strongest if $k_{1}$ is around $k_{p}$, because there $\mathbf{u}\left(\mathbf{k}_{2}\right)$ and $\mathbf{u}\left(\mathbf{k}_{3}\right)$ are already considerably damped. Of course, there is also viscous damping around $k_{1}$, which would counteract the bottleneck effect, but for $k_{1}<\eta^{-1}$ the damping by viscosity $\nu$ is weaker than the damping by the eddy viscosity [26].

For $k \ll k_{p}$ Falkovich [26] calculates the first order correction to the K41 spectrum due to the bottleneck phenomenon and obtains

$$
F^{(2)}(\tilde{k}) \propto F_{K 41}^{(2)}(\tilde{k})\left[1+\tilde{k}^{4 / 3} / \log \left(\tilde{k}^{-1}\right)\right], \quad \tilde{k}=k / k_{p} .
$$

We tried to compare our results for the scaling of the correction term with his prediction $\propto \tilde{k}^{\beta^{\prime}}, \beta^{\prime}=4 / 3$ (apart from the log-correction), but as $k \ll k_{p}$ (i.e., $\tilde{k} \ll 1$ ) has to hold, we can only fit with a very limited number of data points. As in addition for small $k$ the design matrix of the fitting problem nearly degenerates, 
our results strongly depend on the fit range we choose and we can neither verify nor rule out his prediction $\beta^{\prime}=4 / 3$.

The same pileup as for $F^{(2)}(k)$ also appears in higher order velocity moments $\left\langle|\mathbf{u}(\mathbf{k})|^{m}\right\rangle$ with $m>2$, leading to smaller local scaling exponents $\zeta_{m}(k)$ for $k$ near the VSR. Possibly this effect mimicks intermittency corrections to classical scaling in experimental data or simulated data with shorter scaling ranges which in fact would not show up in sufficiently long ISR for large enough Re. This interpretation is also supported by the behavior of the scale resolved intermittency exponents, $\zeta_{m}(k)$, see Fig. 3 above and ref. [12.

\subsection{Log-similarity description of the spectra}

Finally, besides the normalization of the spectra (1) another procedure has been suggested to get an universal description of the experimental data, namely, the log-similarity description [15]. This claims that the logarithmic spectra coincide for different $R e_{\lambda}$, when both the abszissa and the ordinate are multiplied by some function $\beta\left(R e_{\lambda}\right)$, i.e., $\beta \log \left(|\mathbf{u}(\mathbf{k})|^{2} /\left|\mathbf{u}\left(\mathbf{k}_{0}\right)\right|^{2}\right)$ against $\beta \log \left(k / k_{0}\right)$ is claimed to be universal. $k_{0}$ is a wave number which has to be fitted to the experimental data. In [15] $k_{0} \approx 2 k_{p} \approx(6 \eta)^{-1}$ is found. For large $R e_{\lambda}$ it is $\beta=0.9 / \log \left(R e_{\lambda} / 75\right)$ [15. We plot the spectra for our three largest $R e$ in this parametrization, see inset of Fig. 5. As Taylor Reynolds number we simply take $\sqrt{R e}$, because our approximation overestimates $R e_{\lambda}$, see the discussion in section 2. For smaller $R e$ the function $\beta\left(R e_{\lambda}\right)$ behaves quite different, so it is not reasonable to show also the spectra for smaller Re in the plot. The quality of the superposition of the spectra might improve if one readjusted the free parameters of this description.

\section{Summary}

To summarize, we first demonstrated the universality of the normalized moments $F^{(m)}\left(k / k_{p}\right)$ up to $R e=1.4 \cdot 10^{7}$ within our approximate Navier-Stokes solution. Second, the $F^{(m)}\left(k / k_{p}\right)$ can pretty well be described by

$$
F^{(m)}(\tilde{k})=\tilde{k}^{-m / 3} \exp \left(-\frac{2 m}{3}(\tilde{k}-1)\right), \quad \tilde{k}=k / k_{p}
$$

Near peak dissipation an additive correction to the classical scaling $F^{(m)}\left(k / k_{p}\right) \propto$ $\left(k / k_{p}\right)^{-m / 3}$ shows up, which might be explained by an energy pileup around $k_{p}$ due to a viscosity induced bottleneck phenomenon [26]. This bottleneck phenomenon might mimick intermittency corrections to classical scaling in experimental or simulated data with less resolution and for smaller Reynolds numbers. 
Acknowledgements: D. L. thanks B. Castaing, G. Falkovich, Th. Gebhardt, and L. Kadanoff for very helpful discussions and the Aspen Center of Physics for its hospitality. Partial support by the German-Israel-Foundation (GIF), by DOE, and by a NATO grant, attributed by the Deutsche Akademische Austauschdienst (DAAD), is gratefully acknowledged. The HLRZ Jülich supplied us with computer time. 


\section{Tables}

\begin{tabular}{|r|r|r|r|r|}
\hline & $R e$ & $R e_{\lambda}$ & $k_{p} / k_{\min }$ & \# of modes \\
\hline best experiments [1] & $1.7 \cdot 10^{8}$ & 13000 & $\approx 10000$ & $\infty$ \\
best simulations [2] & $\approx 4 \cdot 10^{4}$ & 200 & 5 & $4 \cdot 10^{8}$ \\
REWA [here] & $1.4 \cdot 10^{7}$ & 45000 & $\approx 2000$ & $80 \cdot 3 \cdot 13=3120$ \\
\hline
\end{tabular}

Table 1

In the first two lines $R e, R e_{\lambda}$, the length of the scaling range $k_{p} / k_{\min }$, and the number of contributing modes are compared for the most developed experimental and numerical turbulence, respectively. In the third line we give the same data for the largest $R e$ of our reduced wave vector set approximation (REWA).

\begin{tabular}{|r|r|r|r|r|r|r|}
\hline$\nu$ & $l_{\max }+1$ & $R e$ & $R e_{\lambda}$ & $c_{\epsilon}(R e)$ & $\log _{10}\left(k_{p} / k_{\min }\right)$ & $\log _{10}\left(c_{\eta} R e^{3 / 4}\right)$ \\
\hline $5 \cdot 10^{-3}$ & 5 & 730 & 122 & $46 \cdot 10^{-3}$ & 0.24 & 0.08 \\
$5 \cdot 10^{-4}$ & 7 & $1.05 \cdot 10^{4}$ & 801 & $15 \cdot 10^{-3}$ & 0.97 & 0.95 \\
$5 \cdot 10^{-5}$ & 9 & $1.25 \cdot 10^{5}$ & 3590 & $9.1 \cdot 10^{-3}$ & 1.79 & 1.75 \\
$5 \cdot 10^{-6}$ & 11 & $1.37 \cdot 10^{6}$ & 13600 & $7.0 \cdot 10^{-3}$ & 2.48 & 2.53 \\
$5 \cdot 10^{-7}$ & 13 & $1.40 \cdot 10^{7}$ & 44800 & $6.5 \cdot 10^{-3}$ & 3.29 & 3.29 \\
\hline
\end{tabular}

Table 2

Results from our approximate solutions of the Navier-Stokes equation for various $\nu$. $l_{\max }+1$ is the number of rescaled wave number replica $K_{l}$. The definition of $R e$ is $R e=U_{0} L_{0} / \nu$. Here $L_{0}=\pi / \sqrt{6}$, and $U_{0}=2 u_{1, r m s}$ describes the velocity difference across the outer scale, being determined for each $\nu$ from our numerical solution. $\left(u_{1, r m s}\right.$ depends on $\nu$ and increases from 1.42 to 2.73 for the $\nu$ in the table.) $R e_{\lambda}=u_{1, r m s} \lambda_{\text {Taylor }} / \nu$, as usual. The coefficient $c_{\epsilon}$ is calculated according to eq. (2) . In the last two columns the extension of the scaling range, $\log _{10}\left(k_{p} / k_{\min }\right)$ (found from the numerical solution), is compared with that calculated from eq. (雨), see text. 


\begin{tabular}{|r|r|r|r|r|c|}
\hline$\nu$ & $\zeta_{2}$ & $\zeta_{6}$ & $\left(k_{p} \eta\right)^{-1}$ & $\left(k_{d}^{(2)} \eta\right)^{-1}$ & $\tilde{k}_{d}^{(2)}$ \\
\hline $5 \cdot 10^{-3}$ & 0.656 & 1.997 & 10.2 & 11.8 & 0.87 \\
$5 \cdot 10^{-4}$ & 0.678 & 1.986 & 10.6 & 13.6 & 0.78 \\
$5 \cdot 10^{-5}$ & 0.672 & 1.975 & 9.2 & 13.7 & 0.67 \\
$5 \cdot 10^{-6}$ & 0.669 & 1.994 & 10.5 & 13.3 & 0.79 \\
$5 \cdot 10^{-7}$ & 0.668 & 1.990 & 9.1 & 13.3 & 0.67 \\
\hline
\end{tabular}

Table 3

The fit parameters to our approximate solutions of the Navier-Stokes equation for the same $\nu$ as in table $2 . k_{p}$ is the wave number with peak dissipation, $k_{d}^{(2)}$ the cut off from our fit (5), if we fit the spectrum in the interval $\left[0, \eta^{-1} / 4\right]$. In the last column their ratio $\tilde{k}_{d}^{(2)}=k_{d}^{(2)} / k_{p}$ is given. From the condition $\tilde{k}^{2} F^{(2)}(\tilde{k})$ maximal at 1 , the cut off should be $\tilde{k}_{d}^{(2)}=3 / 4$. 


\section{Figures}

Figure 1: $R e_{\lambda}$ (squares) and $c_{\epsilon}$ (circles) as functions of $R e$. Left ordinate is $R e_{\lambda}$, right ordinate is $c_{\epsilon}$. For large $R e$ the power law $R e_{\lambda} \propto \sqrt{R e}$ holds, for small $R e$ there are deviations, as the dimensionless number $c_{\epsilon}$ in eq. (2) depends on Re. The dependence of $c_{\epsilon}$ on $R e$ strongly resembles the experimental one, cf. [15, 18, 13]

Figure 2: Universal spectra $F^{(m)}\left(k / k_{p}\right)$ for $m=2$ (flatter) and $m=6$ (steeper) for $R e=7.3 \cdot 10^{2}$ (triangles), $R e=1.05 \cdot 10^{4}$ (crosses), $R e=1.25 \cdot 10^{5}$ (squares), $R e=1.37 \cdot 10^{6}$ (pluses), $R e=1.40 \cdot 10^{7}$ (diamonds). The arrows indicate the smallest wave number free of forcing, $k_{\text {min }}$, for the respective $R e$. The smallest wave number of all $\mathbf{k} \in K$ is $0.82 k_{\text {min }}$, see section 1.2 . The dashed arrow labels $k_{\text {min }}$ in the simulation by She et al. [2], the dotted arrow marks $k_{\text {min }}$ in the one of Vincent and Meneguzzi [5].

Figure 3: Scale resolved intermittency corrections $-\delta \zeta_{m}(k)$ for $m=2,4,6,8,10$, bottom to top. $\nu=5 \cdot 10^{-7}, R e=1.4 \cdot 10^{7}$. The fit range is $[k / \sqrt{10}, k \sqrt{10}]$ for all $k$.

Figure 4: Universal energy dissipation rate $\epsilon(k) / \epsilon\left(k_{p}\right)$ versus $k / k_{p}$ for $\nu=5 \cdot 10^{-7}$, $R e=1.4 \cdot 10^{7}$. Also shown are the fits resulting from (5) (dashed) and (6) (solid).

Figure 5: Universal spectrum $F^{(2)}\left(k / k_{p}\right)$ for $\nu=5 \cdot 10^{-7}, R e=1.4 \cdot 10^{7}$. The fits (5) (dashed) and (6) (solid) are compared, both with the same $\zeta_{2}=2 / 3$. Inset: Quality of universality in the log-similarity description for the second moments, see text. The symbols mean $R e=1.25 \cdot 10^{5}$ (squares), $R e=1.37 \cdot 10^{6}$ (pluses), $R e=1.40 \cdot 10^{7}$ (diamonds). On the abscissa we plotted $\beta \log _{10}\left(k /\left(2 k_{p}\right)\right)$, on the ordinate $\beta \log _{10}\left(\left\langle|\mathbf{u}(k)|^{2}\right\rangle /\left\langle\left|\mathbf{u}\left(2 k_{p}\right)\right|^{2}\right\rangle\right)$ with $\beta=0.9 / \log \left(R e_{\lambda} / 75\right)$, cf. [15]. 


\section{References}

[1] H. L. Grant, R. W. Stewart, and A. Moilliet, J. Fluid Mech. 12, 241 (1961); A. L. Kistler and T. Vrebalovich, J. Fluid Mech. 26, 37 (1966); R. W. Williams and C. A. Paulson, J. Fluid Mech. 83, 547 (1977); F. H. Champagne, J. Fluid Mech. 86, 67 (1978).

[2] Z. S. She, S. Chen, G. Doolen, R. H. Kraichnan, and S. A. Orszag, Phys. Rev. Lett. 70, 3251 (1993).

[3] A. N. Kolmogorov, CR. Acad. Sci. USSR. 30, 299 (1941); A. M. Obukhov, CR. Acad. Sci. USSR. 32, 19 (1941).

[4] Z. S. She and E. Jackson, Phys. Fluids A 5, 1526 (1993).

[5] R. Kerr, J. Fluid Mech. 153, 31 (1985); J. Fluid Mech. 211, 309 (1990); A. Vincent and M. Meneguzzi, J. Fluid Mech. 225, 1 (1991).

[6] A. N. Kolmogorov, J. Fluid Mech. 13, 82 (1962).

[7] F. Anselmet, Y. Gagne, E. J. Hopfinger, and R. Antonia, J. Fluid Mech. 140, 63 (1984).

[8] C. Meneveau and K. R. Sreenivasan, J. Fluid Mech. 224, 429 (1991).

[9] S. A. Orszag, J. Fluid Mech. 41, 363 (1970).

[10] S. Grossmann and D. Lohse, Z. Phys. B 89, 11 (1992); Physica A 194, 519 (1993).

[11] J. Eggers and S. Grossmann, Phys. Fluids A 3, 1958 (1991).

[12] S. Grossmann and D. Lohse, "Scale resolved intermittency corrections", Phys. Fluids A (1994), in press; "Intermittency: Recent results from a reduced wave vector set calculation", Physica Scripta (1993), in press.

[13] L. D. Landau and E. M. Lifshitz, Fluid Mechanics (Pergamon Press, Oxford, 1987).

[14] A. S. Monin and A. M. Yaglom, Statistical Fluid Mechanics (The MIT Press, Cambridge, Massachusetts, 1975).

[15] B. Castaing, Y. Gagne, and M. Marchand, Physica D (1993).

[16] D. P. Lathrop, Jay Fineberg, and H. S. Swinney, Phys. Rev. Lett. 68, 1515 (1992); C. Doering and P. Constantin, Phys. Rev. Lett. 69, 1648 (1992). 
[17] D. Lohse, "On the Re-dependence of the Taylor-Reynolds number", Preprint, Chicago 1994; C. Doering and P. Constantin, "Variational bounds on energy dissipation in incompressible flows", Preprint, Chicago 1993.

[18] K. R. Sreenivasan, Phys. Fluids 27, 1048 (1984).

[19] R. Kerr, 1993, private communication.

[20] C. Foias, O. Manley, and L. Sirovich, Phys. Fluids A 2, 464 (1990).

[21] K. R. Sreenivasan, J. Fluid Mech. 151, 81 (1985).

[22] I. Procaccia, E. S. C. Ching, P. Constantin, L. P. Kadanoff, A. Libchaber, and X. Z. Wu, Phys. Rev. A 44, 8091 (1991).

[23] V. L'vov and I. Procaccia, "Extended universality in moderate Reynolds numbers flow", Preprint, Rehovot 1993.

[24] S. Grossmann, D. Lohse, V. L'vov, and I. Procaccia, in preparation.

[25] H. Effinger and S. Grossmann, Z. Phys. B 66, 289 (1987).

[26] G. Falkovich, "Bottleneck phenomenon in developed turbulence", Preprint, Rehovot 1993. 